\title{
Carnets
}

Revue électronique d'études françaises de l'APEF

Deuxième série - 13 | 2018

Corps, rythmes et voix : en/jeux littéraires et artistiques

\section{Créolité et voix de résistance chez Édouard Glissant}

Pour une identité-relation dans le cadre du Tout-Monde

\section{Adelaide Gregório Fins}

\section{(2) OpenEdition}

Journals

Édition électronique

URL : http://journals.openedition.org/carnets/2563

DOI : $10.4000 /$ carnets. 2563

ISSN : 1646-7698

Éditeur

APEF

Référence électronique

Adelaide Gregório Fins, "Créolité et voix de résistance chez Édouard Glissant », Carnets [En ligne],

Deuxième série - 13|2018, mis en ligne le 31 mai 2018, consulté le 10 décembre 2020. URL : http:// journals.openedition.org/carnets/2563; DOI : https://doi.org/10.4000/carnets.2563

Ce document a été généré automatiquement le 10 décembre 2020.

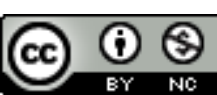

Carnets est mis à disposition selon les termes de la licence Creative Commons - Atribution - Pas d'utilisation commerciale 4.0 International. 


\section{Créolité et voix de résistance chez Édouard Glissant}

Pour une identité-relation dans le cadre du Tout-Monde

Adelaide Gregório Fins

Savoir ce qui dans vos yeux berce Une baie de ciel un oiseau La mer, une caresse dévolue Le soleil ici revenu...

Savoir ce qui sur vos cheveux Hagard étrenne ses attelages Et le sel vient-il de la mer Ou de cette voix qui circule...

Beauté des routes multicolores Dans la savane qui rumine

L'autan plein de mots à éclore Je vous mène à votre seuil...

Édouard Glissant, Poèmes Complets

$1 \mathrm{Au} \mathrm{Xx}^{\mathrm{e}}$ siècle, la re-traversée du bateau négrier d'où s'échappent les cris de douleur et le bruit des chaînes étonne le monde contemporain et fait sortir du silence la part dévastatrice de la domination humaine. De nouveaux récits révèlent alors l'occultation de l'idéologie coloniale, son lot violent de tragédies (Code Noir, Traite), dévoilant ainsi ce que l'homme fait à l'humanité. Constatant par ailleurs une autre catastrophe historique plus récente, le philosophe allemand Theodor W. Adorno (Adorno, 1958) ira jusqu'à se demander si, après Auschwitz, la littérature peut encore se justifier par sa fonction traditionnelle d'enchantement du monde.

2 Il apparaît en effet que la question de l'oppression économique se voit désormais remplacée par les questions relatives à la domination idéologique et coloniale, dessinant 
un véritable changement de paradigme. Il résulte de cette transformation une série d'événements politiques, culturels et sociaux à l'origine des nombreuses transformations de la société ainsi que du pouvoir de l'imaginaire social à l'œuvre dans la littérature.

De fait, avec ces transformations sociales, il s'est ouvert au sein des savoirs scientifiques un nouveau champ de recherche et de questionnement - les «études postcoloniales »comme tentative de penser cet état du monde que furent la colonisation et la décolonisation: " $\mathrm{A}$ la fin $\mathrm{du} \mathrm{xIX}^{\mathrm{e}}$ siècle et au tout début $\mathrm{du} \mathrm{xx}^{\mathrm{e}}$, lorsqu'on disait la civilisation des peuples d'Afrique, on comprenait l'action de civiliser ces peuples et non l'ensemble de leurs manières de vivre et de penser » (Dictionnaire des civilisations africaines, 1968).

4 La violence coloniale constitue le cœur de la problématique contemporaine de la domination, notamment chez Frantz Fanon, Aimé Césaire, Édouard Glissant, Léopold Senghor, Achille Mbembe, Patrick Chamoiseau ou Ashis Nandy, pour ne citer que les plus emblématiques. Partant de la réflexion critique et créatrice de ces auteurs, nous analyserons la colonisation aux Antilles, son passé refoulé, pour essayer de cerner les effets de la domination coloniale sur la mémoire individuelle et collective, et tenter de comprendre le rôle de la subjectivité dans l'imaginaire social.

De fait, ce changement de paradigme nous permettra d'interroger le rôle joué par l'imagination de l'écrivain en rapport avec l'exigence d'une revalorisation de la culture noire, notamment à partir de l'interrogation de Patrick Chamoiseau : «Comment écrire alors que ton imaginaire s'abreuve, du matin jusqu'au rêve, à des images, des pensées, des valeurs qui ne sont pas les tiennes?» (Chamoiseau, 1997).

6 Nous poursuivrons cette analyse en posant la question de la culture et de la langue dans le rapport du sujet à son passé, avec pour perspective l'esquisse d'une ère nouvelle entre l'Afrique, les Antilles et le monde. Il s'agit de revenir à ce contexte de multiethnicité ou d'espace culturel chaotique dans lequel s'entrechoquaient des valeurs venues d'Afrique, d'Europe, d'Asie, de l'Inde et d'Amérique. Dans ce « chaos-monde » (Glissant, 1997), nous suivrons ce tracé opaque de la parole créole d'où surgit une création artistique antillaise qui mobilise autant l'oralité que l'écriture pour nous faire sentir la poétique du paysage et du temps naturel antillais (qui n'est pas le temps linéaire de l'Occident).

7 Ainsi nous convoquerons les notions de négritude et de créolité chez les auteurs cités plus haut et tenterons d'analyser ici les différents points de rencontre de la langue écrite et parlée avec l'éthique et le politique, en partant de l'effort déployé par la nouvelle littérature créole qui invente une langue pour dire et exprimer la violence produite par l'esclavage et la colonisation.

8 En effet, confrontés à l'idéologie de l'impérialisme colonial, porté par l'idéal de progrès des Lumières et la rationalisation à visée universelle, son autorité totalisante, nous questionnerons la langue créole, ainsi que les passeurs de créolité, pour savoir dans quelles conditions la littérature peut encore faire advenir la part subjective des êtres humains libérant leur pouvoir créateur. Pouvons-nous ainsi trouver dans le modèle d'écriture littéraire antillais une portée éthique et politique à visée universelle?

Dans son texte fondateur, Le discours antillais (Glissant, 1997), Édouard Glissant analyse la réalité économique, politique et culturelle des dominés antillais à travers un « discours de méthode » théorique et critique, associant sa pensée militante au réel antillais. Il inscrit sa réflexion dans un héritage "Le marronnage et le refus... l'Ailleurs et le rêve " (Glissant, 1997: 32) et pose la question de l'identité. Entre présent chaotique et projet 
pour l'avenir, ce texte s'adresse à tous les peuples et évoque «le devoir sévère de méditer la fonction des langues, la trame du langage... La pratique de plusieurs langues qu'il faut 'maîtriser'« (Glissant, 1997 :455), attestant que le créole est à la fois langue de soumission et langue de libération.

Il semble nécessaire de revenir à la langue créole, ou plus exactement à une voix française créolisée, dans la mesure où il est impossible de faire table rase du passé colonial. Mais Glissant se trouve ici tiraillé entre la voix européenne, celle du concept ou de l'universel, celle notamment de sa formation intellectuelle à Paris, et la voix créole d'origine, celle de sa singularité qu'il a longtemps refoulée. Le poète, philosophe et romancier creuse ainsi les thèmes littéraires pour faire émerger le refoulé et élaborer une poétique et une philosophie de la relation, voire une éthique de la proximité. S'il pense toujours en poète l'homme dans son milieu, ou si l'on préfère l'être-en-interaction, c'est pour nous conduire vers son concept de "Tout-Monde » (Glissant, 1997) où s'expriment les tensions du multilinguisme.

Glissant s'inscrit dans le «mouvement de la négritude » (Césaire, 1939), un détour par l'Afrique que Frantz Fanon avait accompli à travers la langue et le discours politique (Fanon, 1952, 1985), une œuvre qui s'enracine à la fois dans la créolité et dans le combat pour la libération du peuple noir. Détour accompli aussi chez Aimé Césaire à travers la poétique de la négritude (Césaire, 1939, 1950, 1987), celle des "fils aînés du monde " (Césaire, 1939) et de la lutte pour la reconnaissance du peuple martiniquais où la mémoire douloureuse et la dignité humaine s'entrecroisent. Or, ce moment nécessaire doit être selon Glissant complété par l'idée « marronne » (Glissant, 1958) qui ouvre une perspective d'autocritique, de résistance et de renouvellement.

Nous savons que le terme de «négritude » est introduit par le poète Aimé Césaire, dans la revue contestataire qu'il a créé avec Léopold Senghor, L'Étudiant noir, en 1934. La négritude est ici définie par Césaire comme « la simple reconnaissance du fait d'être noir, et l'acceptation de ce fait, de notre destin de Noir, de notre histoire et de notre culture " (Senghor, 1977). Alors que chez Senghor ce concept intègre les valeurs culturelles de l'homme noir, son auto-affirmation et sa solidarité, sa révolte et sa souffrance contre l'oppression du colon blanc, d'où une définition plus étendue de ce concept: "La négritude, c'est l'ensemble des valeurs culturelles du monde noir, telles qu'elles s'expriment dans la vie, les institutions et les œuvres des Noirs. Je dis que c'est là une réalité : un nœud de réalités » (Senghor, $1964: 9$ ).

13 Le terme de " négritude " va constituer alors un pivot d'ancrage idéologique et esthétique contre la politique française d'assimilation et son mépris pour ce qu'elle appelle le primitif africain et ses descendants des îles d'outre-mer: la relation étroite avec la nature, les croyances populaires, les pratiques magico-religieuses, les nourritures, etc. Face à cet état d'anéantissement de l'autre, celui que l'universel unique des Lumières et du républicanisme français considère comme mineur, l'élite intellectuelle et savante noire fait un retour à ses sources culturelles pour les valoriser comme composantes d'une identité africaine retrouvée. Ce moment de la négritude, porté par Césaire et Senghor, comme prise de conscience de la condition de l'homme noir à partir de ses racines, contre l'hégémonie politique, culturelle, intellectuelle et raciale de l'impérialisme colonialiste européen, est un pilier de résistance intellectuelle noire qui débute à partir de 1931, au sein de la Revue du Monde noir, et où se retrouvent des intellectuels de la diaspora d'Afrique, soucieux de l'héritage commun de la population noire; il trouvera son 
prolongement, quelques années plus tard dans La Présence Africaine (1947), avec la divulgation et les publications des idées, œuvres et manifestes panafricains.

Essentiellement, cette négritude est une voix qui dit l'expérience violente et la révolte des descendants d'esclaves africains en réponse à l'impérialisme colonial. De fait, la voix de cette négritude parle de résistance et décrit sa relation à l'identité, une identité qui revendique le droit à la différence. Césaire fait œuvre de fondation et même de refondation ouvrant les traces marronnes à d'autres écrivains. Ce mouvement de négritude césairien a ouvert la voie à la créolité, en accordant davantage d'attention au caractère chaotique de la vie humaine qu'au concept. Le poète fera ainsi sortir l'individu collectif antillais du vide mémoriel maintenu par la colonisation, mais il ouvrira également le chemin d'un homme nouveau aux Antilles.

Certes, la négritude césairienne a ouvert la voie à la créolité : antillaise, africaine, guyanaise, brésilienne, asiatique, indienne, un "monde diffracté mais recomposé", disent les auteurs de l'Éloge de la créolité (1989) - Jean Bernabé, Patrick Chamoiseau et Raphaël Confiant. Ce manifeste est à la fois un texte militant et politique, qui appelle d'abord à l'union des peuples créoles de l'Archipel caribéen, puis à l'union avec les peuples anglophones et hispanophones. Par ailleurs, ces auteurs plaident pour un régime politique où la pluralité des partisans, confessions et syndicats a toute sa place. Manifeste aussi pour une littérature de fondation qui instaure un nouveau temps. Les auteurs parlent d'un monde dont le poids de la réalité passée se trouve encore inscrit dans le quotidien antillais. La grande force de l'Éloge de la créolité (1989) est d'avoir montré qu'il n'y a pas d'Histoire avec majuscule, mais une pluralité d'histoires ordinaires, intimes, et où seule la connaissance poétique et littéraire porte les moyens d'une résistance, d'une nouvelle conscience et d'une connaissance de soi, qui passent par une « sentimenthèque » (Chamoiseau, 1997) d'où surgissent des lectures fondatrices.

16 Effectivement, ces écrivains trouvent et redécouvrent un lieu et un héritage commun d'écriture : la négritude. Cependant, "plus important que la source est le chemin d'eau ", dit Glissant dans Sartorius. Le roman de Batoutos (Glissant, 1999 :53). Ce chemin est celui qui sert de pont entre l'Afrique et les Antilles pour nous montrer les contours de l'imaginaire des langues et du rhizome où poussent toutes les hybridations transatlantiques. Certes, l'imaginaire antillais et la créolisation doivent beaucoup à la négritude de Césaire, mais si d'un côté nous trouvons chez Glissant une filiation entre africanité et négritude, nous y trouvons aussi une filiation entre créolité, antillanité et américanité. Il est important de rappeler que pour Glissant il n'y a pas d'opposition entre créolisation et négritude. Négritude, africanité et hybridation multiculturelle se mêlent dans le même champ littéraire antillais postcolonial.

17 L'analyse de l'immense œuvre littéraire de Glissant: poésie, essais poétiques, essais politiques, romans, théâtre, passe nécessairement par une pluralité de lectures. L'ensemble fait entendre des voix/voies diverses et met en relation les aspects de la vie créole sous une forme d'écriture de soi soucieuse d'autrui. Cette œuvre littéraire est étroitement liée à sa pensée politique et nous savons que Glissant est un militant politique qui s'associe à des actions en faveur des minorités, des opprimés et des luttes anticoloniales.

18 À suivre l'œuvre de l'écrivain, notamment son roman Malemort, écrit en 1975, Glissant dévoile face au dénigrement des colonisés blancs le réel antillais : un imaginaire oublié, faisant place à un créole recréé qui émerge de la vision intérieure, par et pour l'écriture, à partir de la connaissance et de l'acceptation de soi. Cette vision de soi intérieure est 
nécessaire pour accéder à l'antillanité, et la créolité en constitue le ciment. Une créolité basée sur le mode de l'oralité traditionnelle des esclaves dans les plantations: dictons, contes, chansons, proverbes, comptines, etc. Une créolité qui résiste au système d'écriture élitaire français et qui fait le choix de la parole, le choix de l'enracinement de l'oralité dans la relation concrète des gestes quotidiens. À ce sujet, regardons encore ce qu'Édouard Glissant affirme pour caractériser la personne caribéenne et la créolisation : "Je te parle dans ta langue et c'est dans mon langage que je te comprends " (Glissant, $1969: 53)$.

$19 \mathrm{Au}$ terme de ce premier temps d'analyse, nous pouvons dire que l'esthétique créole glissantienne comporte une pensée complexe qui se bâtit sur la polyphonie, ainsi qu'une hybridation qui représente les voix/voies de résistance à partir de la pensée du marronnage. Le deuxième moment de cette analyse, est consacré à l'écriture 'poétique' littéraire, notamment à partir de la Poétique de la Relation (Glissant, 1990) et du Traité du Tout-Monde (Glissant, 1997), parce que ces œuvres nous invitent à poser ces questions essentielles: comment être soi-même sans se fermer à l'autre? Et dans le même mouvement : comment s'ouvrir à l'autre sans se perdre ?

En effet, la Poétique de la Relation révèle combien la notion d'ouverture à l'autre en même temps qu'à soi-même est essentielle pour résoudre le rapport binaire de la culture et des langues dominantes/dominées. En outre, nous y trouvons une pensée mouvante des cultures composites, cette association rhizomique (Deleuze, Guattari, 1980) des voix plurilingues, écritures, espaces, lieux, autrement dit une forme « archipélique » (Glissant, 1996 : 43) de créolisation qui nous permet de comprendre l'apport glissantien au débat éthique et politique présent dans cette littérature de l'antillanité, terme que l'auteur propose parce qu'il définit davantage l'acte de survie de l'expression orale des îles de la Caraïbe ainsi que son esprit de résistance au système :

La pensée des créolisations : comme inexprimable du rapport des cultures entre elles, avec tant de prolongements inattendus, qui distingue tellement la créolisation des simples hybrides. Mais nous (soit ethnique, sociétal, culturel, continental ou archipélique) ne concevons d'abord pas ces inattendus, qui introduisent aux incertains de la Relation... La créolisation n'est ni l'évidence de cette hybridation seulement, ni le melting-pot, ni la mécanique des multiculturalismes. C'est processus, et non pas fixité. Il y a une alchimie de la créolisation, qui outrecroise les métissages (Glissant, $2009: 64)$.

21 Si l'auteur refuse de s'enfermer dans une représentation monolithique de la négritude (celle des premiers concepteurs de ce terme), il nous recommande de prendre en compte la complexité de la réalité créole. En effet, d'après lui, cet effort pour symboliser ce qui s'est passé et redonner vie et dignité à la voix créole va au-delà de la contestation du peuple des Caraïbes à la colonisation française, il va au-delà de la question du droit.

22 Ainsi, sa définition de la créolité va faire place à l'écriture oralisée de l'antillanité, montrant une identité antillaise qui mêle les tons, les discours et les genres des peuples des Caraïbes, des colons européens, des Asiatiques, des Indiens, des Américains :

J'avais supposé qu'une langue créole, ni un dialecte ni un patois, ni la déformation géniale et agressive d'une langue dominante, est la résultante imprévisible, imprédictible, et fulgurante, dans un temps et dans un lieu, de la rencontre de données linguistiques (lexiques, syntaxes et modes du parler), absolument hétérogènes, avec des résultantes inattendues: que la créolisation des cultures tient de ces divers caractères et non pas de l'essence d'une nature créole. Quand les pays se créolisent, ils ne deviennent pas créoles, à la manière des habitants des îles antillaises par exemple, ils entrent dans l'imprévu consenti de leurs diversités, 
parfois à grand drame... Quand la créolisation s'accomplit, les langues créoles ne tendent plus à disparaître, elles prolifèrent sur et par elles-mêmes (Glissant, 2009 : $65)$. pour objectif de faire advenir une voix qui exprime une identité-rhizome sous la forme de la relation, attestant que l'humanité porte une «identité-relation» :

La créolité n'est pas ce mélange informe (uniforme) où chacun irait se perdre, mais une suite d'étonnantes résolutions, dont la maxime fluide se dirait : « je change, par échanger avec l'autre, sans me perdre pourtant ni me dénaturer ». Il nous faut l'accorder souvent, l'offrir toujours (Glissant, $2009: 66$ ).

Il s'agit chez Glissant d'une pensée et d'une écriture hybride qui créent une véritable relation esthétique, éthique et politique. Nous cherchons à montrer ici comment la littérature glissantienne porte le rêve d'une parole libre et d'une esthétique du divers, qui tourne le dos à l'héritage des Lumières françaises et aux principes universalistes. Dès le départ, Glissant considère que le monde comporte deux formes de pensée : la pensée continentale, qu'il nomme " pensée de système », et la pensée archipélique :

Une autre forme de pensée, plus intuitive, plus fragile, menacée, mais accordée au chaos-monde et à ses imprévus, se développe, arc-boutée peut-être aux conquêtes des sciences humaines mais dérivée dans une vision du poétique et de l'imaginaire du monde... j'appelle cette pensée pensée archipélique, c'est-à-dire une pensée non systématique, inductive, explorant l'imprévu de la totalité-monde et accordant l'écriture à l'oralité et l'oralité à l'écriture (Glissant, 2008 :34).

Une pensée qui dessine l'imaginaire du passé et en même temps un imaginaire qui se fait programme d'action: une manière de penser aussi bien l'identité, la politique, les langues, les cultures et les modalités de la pensée.

Chez Glissant les «histoires lézardent l'Histoire, elles rejettent sur les bords irrémédiables ceux qui n'ont pas eu le temps de se voir au travers des lianes amassées » (Glissant, 1981 : 227). Le fil de ces histoires qui lézardent, nous les trouvons par exemple dans La poétique de la Relation, où Glissant part de ce qui nous pétrifie : la traite d'Africains vers l'Amérique, « dans une mer qui diffracte » (...) « nos barques sont ouvertes pour tous, nous les naviguons ", il s'agit de l'existence d'ici et d'ailleurs, à la fois «enracinée et ouverte » (Glissant, 1990 : 46). Ces histoires ont le pouvoir de l'arrachement à la pensée unique pour nous conduire à la relation, au multiple et à l'autre. Autrement dit, la pensée archipélique est ce qui permet aux humains de sortir de tout espace insulaire pour révéler ce qu'il y a en nous de plus originel : l'altérité.

Par conséquent, l'écrivain revendique et pratique la polyphonie et oppose à la pensée continentale la pensée archipélique, où espace et identité sont étroitement liés : «Ouvrez au monde le champ de votre identité » (Glissant, 1997 : 68). Il s'agit d'une identité-relation mouvante qui assure le dialogue entre les cultures et la créolisation, "c'est cela que j'appelle identité culturelle. Une identité questionnante, où la relation à l'autre détermine l'être sans le figer d'un poids tyrannique » (Glissant, 1981 : 283). Le noyau de La poétique de la Relation comporte trois notions principales: le multilinguisme, la créolisation et l'opacité (métaphorique et philosophique des contes créoles) et qui à travers une écriture particulière et subjective (première personne du singulier), tentent de saisir une pensée archipélique, ce que l'écrivain appelle le divers, afin d'exprimer une résistance éthique à la science universelle et au sectarisme de la racine unique. En effet, l'œuvre présente des voix diverses et des paroles éclatées, notamment à travers des fragments, qui se construisent à partir des traces, où l'oubli va à 
partir du travail de la mémoire créer une éthique de la relation. De fait, la poétique du divers refusera l'identité en termes de racine ou souche unique pour nous faire rentrer dans l'identité-rhizome, racine qui progresse à entrées multiples, seule à pouvoir admettre et reconnaître l'altérité : « Contribuer par les pouvoirs de l'imagination, à faire lever le réseau, le rhizome des identités ouvertes, qui se disent et qui écoutent" (Glissant, 1997 : 246).

Singulièrement, cette altérité dans les textes d'Édouard Glissant nous est transmise d'une part par l'écriture de soi, où se trouvent mis en partage les murmures, les confidences et les silences; d'autre part, l'auteur fait souvent place au lecteur, il y a souvent un « tu » dans ses récits, il y a toujours un renvoi à l'autre, donc une altérité.

Aussi cette pensée archipélique n'est pas un concept mais l'expérience humaine qui donne à voir le divers du Tout-Monde, autrement dit une vision autre du monde, de notre univers qui change et perdure en changeant: "J'appelle chaos-monde... le choc, l'intrication, les répulsions, les attirances, les connivences, les opposions entre les cultures des peuples dans la totalité ... il s'agit du mélange culturel (Glissant, 2008 :30).

31 C'est une pensée qui se veut éthique, car le langage prolonge le cri de l'esclave dans cette écriture, laissant entendre le bruit du monde sensible et sensuel. C'est aussi une écriture qui rejoint le "possible imaginaire» du Traité du Tout-Monde (Glissant, 1997). Si la poétique de la relation nous donne à voir et à sentir le mouvement des vagues englobant et fécond sous forme de spirale, c'est pour que nous puissions comprendre qu'il n'y a pas de poétique sans le corps. A son tour, le Traité du Tout-Monde nous apprend l'alternance de l'ordre et du chaos: on navigue de bord en bord avec les cris des esclaves venant d'Afrique, on pérégrine avec les " pacotilleuses » qui vont d'île en île et tissent la Caraïbe, jusqu'au "pacotilleur de toutes ses histoires réassemblées " (Glissant, 1997: 462-463) qu'est Édouard Glissant. Ces bribes qui s'expriment dans les traces du voyage et des paysages révèlent l'idée d'un lieu commun entre le proche et le lointain, c'est-à-dire une relation où s'expriment tout à la fois la cohésion et la diversité du monde. Nous constatons alors que Glissant nous propose une esthétique des bribes, du Divers (du tout) et de l'Un (celui qui s'arrache à la communauté des Genres et se libère), ainsi qu'une rhétorique du mouvement. Si sa poésie est une forme d'expression, sa philosophie fragile et vivante des archipels constitue une philosophie de la relation et un mode de connaissance de l'homme et du monde. Par ailleurs, il est intéressant de remarquer que Glissant élabore une poétique de la relation avant de construire une philosophie de la relation.

Nous comprenons alors le lien étroit qui unit sa définition de la pensée archipélique à la notion de créolisation; cependant, en nous conduisant vers le divers et le Tout-Monde, Glissant va plus loin que Senghor et fait exploser les limites de la créolité. De plus, nous observons que cette oralité singulière présente dans la négritude, la créolité et l'antillanité portée de générations en générations par l'imaginaire social, se trouve chez Glissant entourée d'une volonté productrice de mémoire collective malgré l'atrocité du vécu colonial qu'elle dévoile :

La mémoire collective est notre urgence: manque, besoin. Non pas le détail "historique " de notre passé perdu (non pas cela seulement) mais les fonds ressurgis: l'arrachement à la matrice Afrique, l'homme bifide, la cervelle refaçonnée, la main violente inutile. Une évidence absurde - où misère et exploitation se marient à on ne sait quoi de dérisoire - et où perceptible pour nous seuls, se joue un drame sans enjeu apparent, dont il dépend de nous qu'il devienne 
bientôt féconde Tragédie. (Mais qu'est tout ceci dans le drame du monde?)

(Glissant, 1969, rééd. 1997 : 187) place de l'imagination individuelle et collective dans le langage et l'histoire humaine. Cet imaginaire social ou culturel s'accomplit soit à travers l'idéologie, soit dans sa dimension utopique, et le philosophe nous incite ainsi à comprendre sa structure conflictuelle. Ricœur propose ici de réfléchir, d'analyser et de mettre en « ordre des significations et des fonctions distinctes » de ces deux modalités de l'imaginaire social, pour élaborer « la fonction intégrative de l'idéologie (...) et la fonction libératrice de l'utopie " (Ricœur, 1986: 426-430). Le philosophe commence par montrer que chacune de ces notions comporte un sens négatif et un sens positif, un "rôle constructif " et un "rôle destructeur ", une dimension constitutive, essentielle, et une dimension pathologique. S’il refuse l'assimilation de l'idéologie à une illusion ou à un mensonge, il refuse également la vision négative de l'utopie comme fuite hors du réel. Ce texte reprend le débat du langage au sein des idéologies, ainsi que des savoirs historiques et scientifiques, et affirme que l'imagination instituante est nécessaire à l'être même de la société. En effet, un groupe social sans idéologie et sans utopie serait sans projet ni représentation de soi. Nous serions alors face à une société sans perspective globale. Ricœur ne nie pas les pathologies inhérentes à l'idéologie (ce qui fait qu'un groupe croit à son identité) mais il pense que l'utopie est « un exercice de l'imagination pour penser un 'autrement qu'être' du social » (Ricœur, 1986 : 427).

Or l'utopie antillaise porte à la fois une fonction d'intégration et une fonction de subversion. Nous pouvons penser ici l'utopie du mouvement de la négritude, de la créolité et de l'antillanité glissantienne dans sa fonction de contestation, nécessaire pour assurer une critique de l'idéologie de l'impérialisme colonial et de l'universalisme. Par contre, l'utopie présente dans l'antillanité peut réduire le coté totalisant de l'idéologie de la négritude, ainsi que son ancrage dans une identité de racine, qui fixe un point et un ordre à partir de l'Afrique. De fait, l'utopie émancipatrice chez Glissant à travers l'identitérhizome, une identité en mouvement comme nous avons vu plus haut, révèle un ailleurs pluriel et ouvert, qui nous enseigne l'apprentissage de l'altérité, et porte la capacité de donner à la communauté antillaise une «identité narrative » (Ricœur, 1983-85; 1990) et 
littéraire qui révèlent les enjeux de l'identité historique et fictive. Ainsi la mémoire collective du peuple des Caraïbes, en intégrant l'utopie comme récit alternatif pris dans des différentes formes langagières traditionnelles, montre que l'imagination ouvre plus fondamentalement sur le monde, dimension qui fait écho à l'existence de la créolité et de l'antillanité. Partant de ce constat, ainsi que de la conception de l'imaginaire comme "vérité métaphorique » (Ricœur, 1975 : 313), nous sommes en mesure de comprendre la place et la fonction de la métaphore poétique dans l'activité productrice de l'imaginaire social et culturel richement présent dans la littérature antillaise.

En guise de conclusion, nous dirons que le parcours d'Édouard Glissant avec ce que l'auteur appelle la créolité, puis l'antillanité comme projet politique et éthique, constitue une façon de créer du divers, divers qui appelle à une conscience globale et à une résistance continue. Plutôt que de parler de l'universel, l'antillanité nous invite à penser la question de la condition humaine, multiculturelle. Une critique de l'universel, qui passe par cette mise en valeur du singulier à travers l'identité-rhizome, et a pour conséquence le pouvoir de s'opposer aux identités rigides nationalistes. Ainsi l'identité peut se définir à partir de l'hybride et de l'hétérogène afin de créer une relation entre les cultures et les sociétés. Il s'agit de nous conduire vers un humanisme de la diversité, où la notion de relation se substitue à celle des droits universels, ce qui nous permet de comprendre le récit du peuple noir déraciné.

Enfin, l'analyse de ces auteurs, ainsi que la lecture de ces œuvres, nous donnent des outils pour comprendre les enjeux du monde actuel. À cet égard, l'œuvre d'Édouard Glissant est primordiale pour nous aider à penser, par exemple, le contexte de la mondialisation, avec ses flux migratoires et ses exodes. En effet, face à la mondialisation actuelle, le projet d'une communauté internationale, ou d'un "Tout-monde » qui reste à construire, nous invite à être attentifs à la notion glissantienne de « lieu » qui n'a rien avoir avec le terme de fixation. Sa notion de lieu remplace celle d'espace conquis et défendu, donc d'exclusion. Il en résulte que cette notion de lieu est à la fois lieu du monde et lieu commun des tous les êtres humains, puisqu'elle permet de déjouer l'opposition de l'Un et du Multiple. Le lieu pour Glissant est un point de passage, donc tous les lieux se rencontrent, pour nous faire embrasser les divers éléments de la globalité sans pour autant tomber dans la tyrannie de la globalisation. Mais par ailleurs, nous devrions également tenir compte de sa pensée de la relation, pensée qui s'oppose au sentiment d'appartenance, si chère aux nationalismes en vogue actuellement. En effet, face aux systèmes dogmatiques de pensée unique qui menacent notre altérité, les auteurs de la créolité nous rappellent l'importance de l'ouverture aux différentes cultures.

\section{BIBLIOGRAPHIE}

ANDRÉ, Jacques (1981). Caraibales. Paris : Éd. Caribéennes

ADORNO, Theodor W. (1958). Notes sur la littérature. Trad. Sybille Muller (1984). Paris : Flammarion. 
BERNABÉ, Jean, CHAMOISEAU, Patrick, CONFIANT, Raphaël (1989, réed. 1993). Éloge de la créolité. Paris : Gallimard.

BADOT, Alain (1993). Bibliographie annotée d'Édouard Glissant. Toronto : Gref.

BALANDIER, Georges \& MAQUET Jacques (1968). Dictionnaire des civilisations africaines. Paris : Fernand Nathan

CÉSAIRE, Aimé (1939, rééd.1956). Cahier d'un retour au pays natal. Paris : Présence Africaine.

CÉSAIRE, Aimé (1950). Discours sur le colonialisme. Suivi de (1987) Discours sur la négritude. (2011)

Paris : Présence africaine.

CHAMOISEAU, Patrick (1997). Écrire en pays dominé. Paris : Gallimard.

CHAMOISEAU, Patrick (1986). Chronique des sept misères. Paris : Gallimard.

CHAMOISEAU, Patrick (1992). Texaco. Paris : Gallimard.

CHANCÉ, Dominique (2002). Édouard Glissant. Un «traité de déparler ». Paris : Karthala.

DESPORTES, Georges (2008). La Paraphilosophie d'Édouard Glissant. Paris : L'Harmattan.

FANON, Frantz (1952). Peau Noire, masques blancs. Paris : Seuil.

FANON, Frantz (1985). Les Damnés de la terre. Paris : La découverte.

GLISSANT, Édouard (1958, rééd. 1997). La Lézarde. Paris : Gallimard.

GLISSANT, Édouard (1969). L’Intention poétique. Paris : Seuil.

GLISSANT, Édouard (1981, « Folio » 1997). Le discours antillais. Paris : Gallimard.

GLISSANT, Édouard (1990). Poétique de la Relation. Poétique III. Paris : Gallimard.

GLISSANT, Édouard (1993, « Folio » 2002). Tout-monde. Paris : Gallimard.

GLISSANT, Édouard (1994). Poèmes complets (Le Sang rivé, Un Champ d'îles, La Terre inquiète, Les Indes, Le Sel noir, Boises, Pays rêvé, pays réel, Fastes, Les Grands chaos). Paris : Gallimard.

GLISSANT, Édouard (1997). Tout-Monde. Paris : Gallimard.

GLISSANT, Édouard (1997). Traité du Tout-Monde. Poétique IV. Paris : Gallimard.

GLISSANT, Édouard (1996, rééd. 2008). Introduction à une poétique du divers. Paris : Gallimard.

GLISSANT, Édouard (1999). Sartorius. Le roman des Batoutos. Paris : Gallimard.

GLISSANT, Édouard (2006). Poèmes Complets (1947-1993). Paris : Gallimard

GLISSANT, Édouard (2009). Philosophie de la relation. Poésie en étendue. Paris : Gallimard.

MBEMBE, Achille (2010). Sortir de la grande nuit, Essai sur l'Afrique décolonisée. Paris : La Découverte.

MBEMBE, Achille (2013). Critique de la raison nègre. Paris : La Découverte.

MÉNIL, Alain (2011). Les voies de la créolisation. Essai sur Édouard Glissant. Paris : De L'incidence.

MOURA, Jean-Marc (1999). Littératures francophones et théorie postcoloniales. Paris : PUF.

NANDY, Ashis (1984). L'ennemi intime, perte de soi et retour à soi dans le colonialisme. Trad. A. Montaut

(2007). Paris : Fayard.

RICCEUR, Paul (1986). Du texte à l'action, Essais d'herméneutique II. Paris : Seuil.

RICÆEUR, Paul (1975). La Métaphore vive. Paris : Seuil. 
RICCEUR, Paul (1983-85). Temps et récit, 3 tomes. Paris : Seuil.

RICÆUR, Paul (1990). Soi-même comme un autre. Paris : Seuil.

RENAUT, Alain (2009). Un humanisme de la diversité. Essai sur la décolonisation des identités. Paris :

Flammarion.

FONKOUA, Romuald (2002). Essai sur une mesure du monde au XX $X^{e}$ siècle. Édouard Glissant. Paris :

Honoré Champion.

SENGHOR, Léopold (1964). Liberté 1, Négritude et Humanisme. Paris : Seuil.

SENGHOR, Léopold (1977). Liberté 3, Négritude et Civilisation, Paris : Seuil.

\section{RÉSUMÉS}

Nous questionnerons la langue créole ainsi que les passeurs de créolité - Frantz Fanon, Aimé Césaire, Édouard Glissant, Léopold Senghor, Achille Mbembe et Patrick Chamoiseau - pour savoir dans quelle mesure la littérature peut aider les subjectivités humaines à libérer leur pouvoir créateur. Cette analyse nous permettra d'interroger le rôle de la voix/voie de l'écrivain et sa force de résistance. Nous essaierons ainsi d'analyser les différents points de rencontre de la langue avec l'éthique et le politique pour dire et exprimer la violence produite par l'esclavage et la colonisation.

Édouard Glissant évoque dans Le discours antillais (1997) la nécessité de revenir à la langue créole, ou plus exactement, à une voix française créolisée. De là l'élaboration d'une Poétique de la Relation, qui est également une éthique de la proximité, articulée autour du concept de « Tout-Monde » où s'expriment les tensions du multilinguisme et le passage infini entre l'identité et la différence.

We will question the Creole language as well as the Creole smugglers - Frantz Fanon, Aimé Césaire, Édouard Glissant, Léopold Senghor, Achille Mbembe, Patrick Chamoiseau, Edouard Saïd to know to what extent literature can help human subjectivities to release their creative power. This analysis will allow us to question the role of the voice / path of the writer and his strength of resistance. We will try to analyze the different meeting points of language with ethics and politics to express the violence produced by slavery and colonization.

Édouard Glissant evokes in Le discours antillais (1997) the need to return to the creole language, or more exactly, to a creolized French voice. Hence the elaboration of a Poetic of the Relationship, which is also an ethics of proximity, articulated around the concept of the "All-World" expressing the tensions of multilingualism and the infinite passage between identity and difference.

INDEX

Mots-clés : colonisation, négritude, créolité, identité-rhizome, résistance-éthique

Keywords : colonization, negritude, creolity, identity-rhizome, resistance-ethics 


\section{AUTEUR}

\section{ADELAIDE GREGÓRIO FINS}

Lettres Sorbonne Université/Univ. de Coimbra

gfins.adelaide[at]gmail.com 